\title{
Real Time Monitoring for the Most Vulnerable: UNICEF's Experience in Uganda
}

\author{
Matthew Cummins and Barbara Huddleston
}

\begin{abstract}
This article reviews two Real Time Monitoring (RTM) initiatives in Uganda that utilise RapidSMS technology. mTrac uses mobile phones to transmit local health information, including disease surveillance, malaria treatment and drug stocks. This electronic-based reporting system replaced a paper-based one, enabling actionable information to be delivered more quickly. Its early success in Uganda suggests that similar systems could be introduced elsewhere to achieve cost-savings and more efficient monitoring of key supply and demand health indicators in vulnerable areas. U-report offers young Ugandans a chance to voice their opinions on pertinent issues by giving them access to a free SMS service through which they can send in text messages, respond to polls, and receive factual information and results. Although the objective is to empower all youth, $U$-reporters often tackle issues that address the needs of vulnerable children and communities. The ability to target polls by regions is an exciting feature, and further research is warranted to better understand U-report's sampling potential.
\end{abstract}

\section{Introduction: the monitoring challenge}

UNICEF management has a strong interest in Real Time Monitoring (RTM) of social indicators, as a contribution to the Global Pulse launched by the United Nations SecretaryGeneral following the global food and financial crisis. Monitoring mechanisms exist for most indicators of interest, but for many of these indicators, monitoring is too infrequent or reports are too late to be of use when immediate responses to unexpected shocks are called for.

To address this challenge, UNICEF has been encouraging its country offices (COs) since 2008 to experiment with the use of innovative and cost-effective information technologies that could provide relevant and timely information for indicators reflecting the health and welfare status of the most vulnerable, and trigger appropriate and timely responses.

This article reviews two RTM initiatives in Uganda: mTrac and U-report. Both are implemented by the Technology for Development (T4D) programme in the UNICEF Uganda CO and utilise RapidSMS technology.
The study included field visits to locations where U-report and mTrac activities could be observed in operation.

\subsection{Development of the RapidSMS tool}

RapidSMS is an Open Source framework for rapidly prototyping and developing software applications using the Short Message Service (SMS) (or text messaging) function of basic mobile phones and a web interface for information management and analysis. RapidSMS was originally developed by UNICEF's Division of Communication together with graduate students from Columbia University in 2008, who jointly won first prize in USAID's 2008 global innovation competition for the RapidSMS tool they deployed in Malawi to monitor the health of children in danger of malnutrition. RapidSMS is now used by hundreds of individuals, companies and organisations across the globe for a wide variety of purposes.

RapidSMS was designed to be customised for the challenges confronting governments, multilateral agencies, NGOs and development practitioners, including working effectively 
despite geographical remoteness of constituents, limited infrastructure, such as roads and electricity, and slow data collection (due to paper-based recording systems, slow courier systems, etc.). The framework was created by programmers working directly on actual project implementation with end users and not by an isolated software firm working from specifications. The resulting software is practical, flexible and simple enough to meet real world needs. Moreover, RapidSMS is not constrained to any particular kind of mobile device; any mobile phone that can send and receive text messages can interact with RapidSMS technologies.

To date, RapidSMS has been customised and deployed with diverse functions, including remote health diagnostics, nutrition surveillance, supply chain tracking, local food price monitoring, registering children in public health campaigns, and notifying of job opportunities, among others. Early applications included monitoring food supplies in Ethiopia and tracking child malnutrition trends in Malawi. RapidSMS was introduced to UNICEF Uganda at the behest of the newly appointed representative, Dr Sharad Sapra, who had previously been Director of UNICEF's Division of Communication. The software has also been deployed in UNICEF COs in Kenya, Mauritania, Nigeria, Rwanda, Senegal, Somalia and Zambia.

\section{Overview of UNICEF Uganda's T4D programme}

\subsection{Role of T4D in Uganda}

UNICEF Uganda's T4D programme is

innovating technologies and new ways of working to support the CO's programmatic goals to keep children alive (health), safe (social protection) and learning (education). The T4D unit develops products on both a supply- and demand-driven basis, pursuing novel ideas that have the potential to cut costs and maximise efficiency for UNICEF programmes, as well as responding to requests of programme managers for technological help on specific initiatives. In addition to serving programmatic needs, T4D innovations address some of the main challenges facing Uganda, which include: (i) obtaining more timely and accurate data; (ii) improving the quality of services (schools, health centres, police and justice systems, etc.); (iii) increasing access to services, especially in remote rural regions and underserved urban areas; and (iv) increasing meaningful participation of young people in national development.

T4D originally grew out of the vision of UNICEF leadership at the country level, and it is now funded by both core operational funds and contributions from all of the different programmatic areas in the CO. Besides mTrac and U-report, UNICEF Uganda is taking advantage of the high rate of mobile phone penetration in the country (about one-third of the population) by developing a number of other unique real-time reporting applications. Four distinct technologies are currently being developed together with the government and development partners. These include: (i) mobile phones and RapidSMS for data and information gathering and sharing (e.g. mTrac, U-report and EduTrac); (ii) use of mobile phones linked to a web interface (e.g. for birth and death registration and Rapid Family Tracing and Reunification); (iii) use of customer relationship management software for call-in services such as Child Helpline; and (iv) computer-based communication systems for connecting communities, increasing citizen participation, tracking aid flows and improving service delivery (e.g. Information Kiosks for Youth Centres and DevTrac). A brief summary of these different initiatives is provided below (Note: mTrac and U-report are intentionally omitted since they will be discussed in detail in subsequent sections).

\subsection{Summary of current T4D initiatives in Uganda}

- EduTrac: This school monitoring system targets improvement of key education indicators, such as teacher absenteeism, availability of textbooks and functionality of water points and latrines, by collecting routine data from head teachers, school management committees and youth organisations via SMS. After a round of tests in 2011 , the system is being rolled out in 14 districts and is expected to cover over 1,000 schools by the end of 2012 .

- Birth Registration: Through a public-private partnership with Uganda Telecom and the Ugandan Registration Services Bureau, a web and mobile-based system was developed to allow health workers and local government officials to report births, with simple verification and printing of certificates at the sub-district level. In 2012 the system will be 
used to register births as part of a social cash transfer programme that targets two million people, with national scale-up to follow.

- RapidFTR: Developed for Family Tracing and Reunification activities, aid workers collect and share information via mobile phone about children and families in emergency situations. This system has been used in Uganda during joint simulations with UNHCR, Uganda Concern for Rural Children (UCRG), Save the Children and World Vision.

- Child Helpline: UNICEF Uganda is supporting the establishment of a national Child Helpline (available to all at a toll-free number), which will direct calls to regional call centres that will refer child abuse cases to local child protection agencies and case workers, or provide information in the case of other types of calls. Abuse cases will be tracked for completion, and alerts generated to ensure follow-up.

- Information Kiosks for Youth Centres: The information kiosks consist of rows of three to six computer screens and keyboards placed along a wall in a community or youth centre that are linked to a solar-powered battery and a central server from which informational material can be downloaded and viewed. The content includes both downloads from the internet and materials that have been prepared specifically for dissemination through the kiosks. These kiosks are a secondgeneration version of the Digital Drums, and have been developed using very low-cost materials and simple construction techniques suitable for widespread replication in remote areas.

- DevTrac: The objective of DevTrac is to monitor and map the development of Uganda. Through an online map, national data and real-time information on development projects are posted and visualised in order to be used for analysis, accountability and advocacy. This knowledge-sharing tool is currently used by UNICEF to monitor development investments but will be expanded to other donor and government partners during 2012.

\section{Potential of T4D to better respond to the needs of the most vulnerable}

Uganda has made good progress over the past decade in reducing poverty and vulnerability. From around 44 per cent a decade ago, the share of the population which is now classified as poor dropped to 27 per cent in 2009, according to data maintained by the World Bank. Moreover, when the national poverty line is used, the figure is only 7 per cent (8 per cent in rural areas and 2 per cent in urban areas). Viewed from an alternative perspective, however, rural people comprise 86 per cent of the total population, and for many, opportunities to improve their standard of living remain limited. Youth represent a disproportionate share of the total, and among them, HIV/AIDS orphans are among the most vulnerable. Women of child-bearing age without access to adequate ante- and post-natal health care services are another highly vulnerable group. Victims of child abuse and disabled persons are other vulnerable groups that require targeted services and special attention.

\section{T4D offers several pathways by which UNICEF} can support the government of Uganda in reaching out to the most vulnerable segments of the population more effectively. First, the use of RapidSMS tools to gather real-time information about the performance of health and educational facilities can improve service delivery and reach the most vulnerable more effectively with relatively little expenditure of time and effort and minimal financial cost. In the case of mTrac, for example, the intention is to increase the effectiveness of Uganda's health services by ensuring that all health facilities and Village Health Teams (VHTs) are being perceived by the population as effectively serving their needs and adequately stocked with anti-malarial drugs at all times. By improving access to these drugs, it is expected that the most vulnerable will be able to obtain timely treatment, which is especially important within the first 24 hours of onset. Anecdotal reports suggest that the ability of VHTs to make anti-malarial drugs available is resulting in an observable reduction in child mortality in villages where they are working (e.g. mTrac data from September 2012 showed that stock-out rates among VHTs was 2.2 per cent, which was much lower than health facilities).

Like mTrac, EduTrac is meant to provide information that should improve the delivery of primary education services. Though it cannot be stated with certainty, it is likely that the schools where service is currently poorest are those serving the poorer and more vulnerable segments of the rural population, so reducing 
teacher absenteeism and ensuring supplies of textbooks and safe water will disproportionately benefit the poor.

U-report has the overall objective of empowering young people and does not specifically target the most vulnerable, although the polling tool does allow for targeting questions to individuals in vulnerable situations. Additionally, specific issues tackled by U-reporters often address the needs of vulnerable children. Examples of recent polls of this nature include the poll on the reasons for high dropout rates for primary school children in remote areas, which eventually led to the 'Back to School' initiative, as well as the poll on the effectiveness of Uganda's child protection services in meeting the needs of victims of child abuse.

Besides RapidSMS, other T4D technologies also benefit the most vulnerable. For example, mobile phone registration of births and issuance of birth certificates is expected to make it easier to identify and locate vulnerable children and provide them with needed help, and registration of families and children separated from each other in conflict and post-conflict zones has already made it possible to reunify many of them rapidly and at relatively low cost. Moreover, the Child Helpline facilitates the provision of child protection services to victims of child abuse, while Information Kiosks for Youth Centres offer distance learning opportunities to young people that may not otherwise have such access.

From this brief summary, it is clear that T4D offers many possibilities for enabling UNICEF Uganda to achieve its objective of reaching the most vulnerable. It has been designed to reduce costs and increase the effectiveness of different programmes, and appears thus far to be highly successful in doing so.

\section{An in-depth review of two RapidSMS applications \\ mTrac \\ Origins}

The health sector was one of the first to receive T4D attention in Uganda, mainly because the Ministry of Health $(\mathrm{MoH})$ was receiving many complaints about shortages of medical supplies at its health facilities. In 2010, UNICEF Uganda invested in the development of an information technology application that was designed to provide timely surveillance data to managers at the district and national level, while crowdsourcing reports of service delivery complaints from an anonymous SMS hotline in order to improve response and strengthen accountability. The previous cumbersome and slow paper-based reporting system was replaced with a RapidSMS system that made it possible for staff at health facilities to use their own mobile phones to report on stock levels and usage rates for antimalarial medicines, with data made instantly available via SMS and a web-based Dashboard to government managers. This initiative - called mTrac - was initially piloted in a few selected districts with support from the Foundation for Innovative New Diagnostics (FIND); it is now being scaled nationally by the $\mathrm{MoH}$, with support from DFID, UNICEF and WHO.

\section{mTrac today}

The mTrac system was launched nationwide in November 2011. By March 2012 it had been rolled out to approximately 1,000 health facilities in 28 districts in Central Uganda, and it is on track to cover all 5,000 health facilities in 112 districts by March 2013. mTrac uses RapidSMS to accelerate the submission of community and health facility data, including disease surveillance, malaria treatment and drug stock indicators. The electronic monitoring initiative has two main components: (i) strengthening the Health Management Information System by providing real-time disease surveillance, antimalarial drug stocks and logistics information; and (ii) engaging the larger stakeholder community to report service delivery bottlenecks, to generate dialogue and calls for action where failures occur, and to strengthen accountability and governance around timely provision of antimalarial drug stocks. The $\mathrm{MoH}$ is taking full ownership of the system, with data being integrated into other national systems.

\section{Quality}

mTrac uses various methods to verify what is reported by the health facilities, including information provided on the anonymous mTrac hotline, independent reports from VHTs and U-report polls, to help identify areas which need further follow-up and support. Stock-outs of artemisinin-based combination therapy (ACT) at health facilities have steadily decreased during project implementation: 25.2 per cent of reporting facilities indicated a stock-out during week 29, which fell to 14.9 per cent by week 39 . 
Improving the transparency and accountability of the health services system in the country has been a government priority for the past two years, and arrests have begun to be made where district health officials and private medical practitioners and pharmacies were found to be siphoning off medical supplies intended for distribution through government facilities. Between March and June 2012, 524 high priority cases were investigated by the State House Medicines and Health Services Delivery Monitoring Unit, with 362 cases successfully resolved. For as long as this attitude prevails, there is reason to believe that the quality of actionable information will be reasonably good.

\section{Inclusivity}

mTrac is intended to improve access and availability of health services, and to ensure that anti-malarial treatment is available for all Ugandan citizens. At present, the leakage of drugs from the public health system into private clinics and pharmacies means that only those who can afford to pay get access to treatment. The objective of mTrac is to introduce more transparency and accountability into the health system, empowering district health officials and their national managers with real-time, actionable information. This includes the drug supply system, reducing leakage and making supplies more readily available at health facilities throughout the country. This will increase access of vulnerable people to the drugs and motivate them to go to health facilities to obtain treatment.

\section{User acceptance}

The mission had the opportunity to interact with mTrac stakeholders on the occasion of the First Data Users Conference organised by the $\mathrm{MoH}$ during 14-18 May 2012 in Kampala. In addition to participating in several panel sessions and hearing presentations on mTrac and the Ministry's Health Management Information System, the event provided the opportunity for one-on-one discussions with key individuals, including the mTrac focal point in the $\mathrm{MoH}$, the WHO representative on the Technical Working Group for mTrac, and a District Health Monitoring Officer responsible for implementing mTrac in the field. Conversations with these individuals, as well as with District Health Officers met during the field visits, revealed uniform appreciation for the new system, which not only provided information in a timelier manner, but also provided it in a more accessible, computer-based form. Difficulties encountered and suggestions made were almost entirely of a technical and correctible nature, and of a sort that would be expected during the rollout phase of any new reporting system.

\section{Costs}

The short-term, largely capital investment costs of rolling out mTrac amounted to roughly

US $\$ 4.5$ million. This covered the development of the software, the purchase of computers and internet modems for each of the 112 districts in the country (at the time of writing), the setting up of the hotline to handle complaints and emergency alerts, and the training and supervision of staff, the latter of which was the largest up-front investment. Donors, including DFID, funded the first phase of the project, which is expected to last approximately one year.

In contrast to phase one, the costs of phase two are expected to be minimal, with the $\mathrm{MoH}$ assuming full financial responsibility. Specifically, recurring costs should be restricted to operating the toll-free hotline line (e.g. the costs for maintaining the SMS service along with staff salaries), providing internet connection in the 112 districts and maintaining/upgrading the software, as necessary, largely through in-house technicians. The major investment of mTrac has been training, which will not be a recurring cost since the same staff that previously filled the paper information reporting sheets will be implementing the new, electronic system, and they will have already received training during phase one. Also noteworthy is the fact that this new electronic reporting platform is expected to secure immediate cost-savings for the $\mathrm{MoH}$, since mTrac is replacing the previous system that was both laborious (time-intensive) and expensive (physically shipping reporting documents from village health centres to district centres and then on to Kampala).

\section{Sustainability}

The objective is to hand mTrac over to the $\mathrm{MoH}$, which will assume full responsibility for maintaining the standardised reporting system following the conclusion of the first phase. This is likely to be highly sustainable due to the strong ownership of the initiative, its affordability and the institutional arrangements. 
First, country ownership is rock solid. As noted by Dr Mukooyo, who is the counterpart in charge of overseeing mTrac within the $\mathrm{MoH}$, the technological platform was developed as a response to a core need of the $\mathrm{MoH}$ and not a donor-driven or externally imposed initiative. While the system was recognised as a strategy to improve the performance of the $\mathrm{MoH}$ to respond to diverse health needs, especially in remote areas, it was both identified and developed by the government and will be 100 per cent operated by the government at the end of phase one.

Second, all of the initial, heavy investments are being borne during the first phase of the project, with the second phase expected to achieve significant cost-savings. Donor support was, indeed, pivotal to getting mTrac launched. However, once fully operationalised across the country, the ongoing recurring costs to maintain the electronic system will be far cheaper than those under the previous, paper-based reporting system, which incurred high labour and transmission costs, due to the fact that all forms had to be filled out manually on a weekly basis, delivered in paper form to the district health office and then reported to $\mathrm{MoH}$ headquarters in Kampala. Additionally, recurrent costs such as internet support to districts can be shared among a larger pool of development partners, some of whom are currently funding similar items for project-specific needs. Moreover, the cost-savings are expected to be safeguarded within the Health Services Section of the $\mathrm{MoH}$ (where mTrac is situated) and channelled toward priority responses that are detected by the system (e.g. supplying additional medicines/ vaccines in deficit areas or delivering life-saving services/treatments in emergency situations, such as a cholera outbreak).

Third, in line with the recent government drive toward decentralisation, mTrac serves as an effective sub-national service delivery tool for one of its priority sectors (health). In practice, mTrac links community health centres via VHTs (some 5,000 across the country) and public health facilities (some 8,000 across the country) to their respective district-level health units (112 across the country) and ultimately to the $\mathrm{MoH}$ headquarters. Such a modus operandi offers twoway advantages: not only does mTrac feed realtime surveillance and supply information directly to the $\mathrm{MoH}$ at a centralised level to inform national policymaking and budget processes, but it also enables headquarters to respond to remote, localised issues that may arise (e.g. supply shortages, personnel shortcomings or disease outbreaks), thus strengthening the presence of the state (and its accountability) to areas that were previously disconnected.

\section{Replicability}

National situations will dictate whether a system such as mTrac should be considered as a viable option. However, the limited capacity of the government of Uganda across parts of its territory is characteristic of many countries where UNICEF works, so Uganda's experience should be indicative of whether a system such as mTrac can be adapted elsewhere. The early success of the initial rollout suggests that such a system could be introduced in many countries to achieve both cost-savings and more efficient and accurate monitoring of key supply and demand health indicators in vulnerable areas.

In specific terms, mTrac's replication capability is underscored by low start-up and recurring costs, the relative ease in adapting the technology and its ability to mainstream decentralisation efforts. In terms of costs, as discussed earlier, the rollout of mTrac cost under US $\$ 6$ million. While the overall cost of introducing this new health monitoring system can be expected to vary, the initial up-front investment is not prohibitive, and most governments would be likely to be able to receive donor support to cover a significant portion of the start-up expenses. Moreover, for countries that would be switching from paperbased tracking and monitoring systems to mTrac's electronic platform, cost-savings are likely to be generated during the operational phase, making this system financially viable over the long term. Given the proliferation of mobile phone use, even in some of the most remote parts of the world, adaptation of the technology to implement mTrac also appears manageable in virtually all environments.

Regarding decentralisation, many governments have undergone comprehensive decentralisation programmes in Asia, Eastern Europe, Latin America and some parts of Africa. Uganda's experience demonstrates that the mTrac platform is capable of offering a standardised reporting procedure that can be implemented routinely by existing health facilities at district 
or regional levels and by local health units at the community level, thus serving as an effective decentralisation tool. An important aspect of Uganda's success has been the wholehearted commitment of the $\mathrm{MoH}$ to the new system, and such commitment would be a necessary precondition to success elsewhere.

\section{Findings and recommendations ${ }^{2}$}

mTrac users expressed strong satisfaction with the system. Although it had been operational for less than two months at the time of the mission, all those interviewed found it easy to use, and District Officers found that the system provided easy accessibility to reports. District Officers also appeared quite willing to train, supervise and motivate the health workers who are required to send in the SMS reports.

Some difficulty in assembling information, which is now more detailed than previously, was commented upon by users in the health facilities. Also, intermittent access to the internet, and the difficulty of extracting data from the Dashboard and analysing it, were issues raised by District Officers.

It appears that the $\mathrm{MoH}$ is well prepared to take over management of the system in the second phase of the project, and it is anticipated that the cost-savings generated by the elimination of the previous paper-reporting system will be ample to cover the costs of training users and maintaining the online database.

VHTs are about to be trained to provide community-level information on incidence of disease, drug supplies and distribution of drugs directly to the district Dashboard. This will supplement and help to verify information coming from health facilities. It was reported anecdotally that there had been a significant reduction in child mortality in locations where VHTs had been active, apparently due to the timeliness with which treatment was given. While there were mixed views about the effectiveness of VHTs, at the request of the $\mathrm{MoH}$, UNICEF is exploring the costeffectiveness of converting them into salaried government workers as a means of increasing their motivation and commitment to the work.

The Nutrition Department in the MoH observed that there had been a significant decline in the frequency with which data on nutrition indicators were collected since UNICEF withdrew its support in 2009. They felt that if mTrac could be used to fill this gap, this would be of great value for policy and strategy planning.

Specific suggestions for further tailoring mTrac to meet the immediate needs of the district-level health monitoring system include:

- Building more artificial intelligence into the Dashboard by incorporating a pre-formatted report writing function with a set of automated reports (e.g. distribution of stocks - where there are surpluses and deficits);

- Finalising integration between mTrac and the DHIS2 software platform of the $\mathrm{MoH}$ so that District Officers can carry out their own analysis of the data;

- Gradually extending the system to cover additional diseases and drugs as well as periodic information on nutrition indicators, particularly for children under five;

- Capitalising on VHTs to the maximum extent possible, including their active use of the mTrac hotline to report any health service problems that are affecting their communities;

- Conducting a formal survey of VHTs - their motivations, community perceptions, what the indicators say, etc. - to determine whether the reported impact on child mortality is statistically significant;

- Considering integrating the quarterly household register maintained by VHTs into mTrac;

- Exploring the possibility of expanding mTrac to support existing electronic medical records in Uganda, such as OpenMRS, which VHTs could use to register and track patients.

\section{U-report \\ Origins}

U-report was conceived as a social monitoring system using RapidSMS. It offers young Ugandans a chance to voice their opinions on issues that they care about by giving them access to a free SMS service through which they can send in text messages, respond to polls, and receive factual information and poll results. Priority issue areas for UNICEF Uganda are social policy, health, child protection and education, but the system allows for exploration of any issue of concern to young people. 
The system was piloted in collaboration with the Uganda Scouts. UNICEF Uganda developed the software application and built a web platform for managing communications between the social monitors, called U-reporters, and the central managers, and the Scouts recruited the reporters and disseminated and discussed the information gathered on the Scout radio programme.

\section{$U$-report today}

Based on the results of the pilot, the opportunity to partner with U-report was extended to other NGOs, and nine currently participate. Following a media-based recruitment campaign and recruitment activities of the partner NGOs, the system was launched in May 2011. Individuals who register to become U-reporters fill in profiles that help categorise the information they provide by age, gender, youth group and location. At the end of 2012, there were already more than 170,000 U-reporters across Uganda, with the average age of a U-reporter being 24. Each week two or three SMS polls or questions are sent to all U-reporters asking for factual information, experiences and/or opinions on a given issue, and the data is mapped to each district and compiled nationally. In return U-reporters receive the results as well as useful facts for action and advocacy related to the particular issue.

U-report has multiple uses, such as raising awareness on specific issues, community-led development and information sharing, including with parliamentarians, as well as policy advocacy. To date over 200 polls have been posted on the U-report website (http://ureport.ug/). Results of U-report polls are featured in weekly television and radio shows as well as in newspaper spreads. In addition, parliamentarians have begun to receive U-report updates that they can use in their legislative work, and they regularly answer questions that are sent in by U-reporters from around the country. In fact, every member of the Ugandan parliament is now part of the U-report programme, and over 100 parliamentarians have expressed interest in using U-report to improve their ability to remain abreast of citizen's views in their constituencies or to answer questions posed to them by U-reporters in the national press or on television. There has also been a U-report conference that brought U-reporters face-to-face with parliamentarians and government officials. The system is still growing and adjusts to the demands of stakeholders as required, so what is written today about U-report could quickly become outdated.

\section{Quality}

Trustworthiness of the information being provided is an issue that the U-report managers and NGO partners constantly manage. Most of the information is provided in the form of responses to polls. To ensure data quality, the system only allows reporters to submit one response in order to avoid any skewing of the data through repeated answers. Additionally, only those U-reporters who are asked the question can answer it; messages received from numbers that are not asked the question are excluded from the poll results, which helps to keep the data clean. Unsolicited data currently comprises 7-10 per cent of the total number of messages received by the system, and UNICEF Uganda is currently working with IBM on methodologies to automatically classify and analyse the information. This kind of quality control is possible at the current level of operation, and through innovation and improved technological methods, the managers are putting in place systems to ensure that this can be managed at scale.

\section{Inclusivity}

U-report is non-discriminatory in design. Anyone who fills out the registration profile can become a U-reporter. Recruitment activities are targeted at youth (defined as anyone up to the age of 30), and there are deliberate efforts to sign up U-reporters in remote areas through promotional activities of NGO partners working in more marginalised parts of the country.

Moreover, in schools and villages it often occurs that children who want to make their voice heard give their messages to adult patrons, who send in an SMS on behalf of the child. This type of group reporting is encouraged so that young persons who do not own mobile phones are able to participate as U-reporters; however, it is currently not possible to identify group messages, which are counted as just one person.

\section{Relevance and user acceptance}

U-report is very well liked by those interviewed for this study, and there are high hopes that it may become a genuine vehicle to allow the voice of Ugandan youth not only to be heard, but also to make a difference in the social and political life of the country. Anonymity is an important 
concern. U-reporters want to express their opinions frankly, even on issues that are highly sensitive. Nevertheless, there are some worries about registering actual names for fear that authorities might be able to obtain a warrant and search the database in the case of sensitive or controversial information being revealed through U-report. For this reason, there is an option to register an actual name, a nickname or no name. U-reporters are also informed during registration that their names/nicknames could be used in the media.

Another important point is the extent to which U-report really will allow the voices of young people to be heard. Individuals who send in messages on their own initiative have no way of knowing whether the message is read, much less acted upon, unless they receive a response. While individual responses are not the norm, there are special question and answer sessions where experts are on hand to answer SMS questions submitted by U-reporters. Poll topics are currently determined by the system managers, their NGO partners (who represent the interests of hundreds of thousands of young people nationwide) and, in some cases, the reporters themselves. However, U-reporters do not have control over the selection of each topic or poll.

Despite these potential drawbacks, U-reporters are encouraged by the fact that several of their polls have resulted in concrete actions being taken as well as by the interest of parliamentarians in using the system to reach out to their constituents. It should be noted that while the system is designed to be social, not all opinions can possibly be shared via U-report, although submitted SMSs are sometimes used as poll questions, alerts or responses.

\section{Costs}

UNICEF is the sole funder of U-report, which had a budget of approximately US $\$ 500,000$ for 2012. This overall sum covers staff time (a fulltime manager, two part-time programmers, one youth participation consultant and three data analysts), recruitment campaigns through mechanisms such as radio commercial spots, television shows, and materials for partner organisations to carry out awareness and training activities (the average enrolment cost is roughly US $\$ 12 /$ person), and the cost for sending and receiving the messages (the average cost to send an SMS to all U-reporters is US\$1,700). It is important to note that the system functions on the basis of volunteer contributions of both the U-reporters and the NGO partners, which are driven by the incentives that are offered to youth via personal empowerment and having a voice, and the benefits the NGOs derive from mobilisation of responses on issues that are of interest to them.

\section{Sustainability}

Sustainability is one of the key questions about U-report. Although nine NGO partners support U-report by participating in decisions about poll questions and recruiting U-reporters, UNICEF Uganda remains ultimately responsible for designing, implementing and managing the technology. There is little doubt that UNICEF's central role has been necessary, reflecting the complicated nature of a reporting system that represents not only technological innovation, but also innovation in terms of the human dimensions of systematically channelling the voices of a large number of youth in a manner that is socially and politically neutral, yet of sufficient interest to attract the attention of those whose action is needed to address the issues being raised.

The intention of the Uganda CO is to slowly turn control over to partner NGOs via a consortium. This approach has benefits as well as challenges. On the one hand, a consortium approach enables the system to be operated by end users as well as to inform policymakers of different issues. On the other hand, a consortium of NGOs could potentially be captured by one of the stronger partners or lead to disagreement in terms of messaging priorities and advocacy activities, and undermine its usefulness. There are also questions surrounding the capacity of a consortium of NGOs to manage the technology and the polling process as well as to adequately fund the recurring costs.

Another possible arrangement for the future management of U-report could include housing the system within the donor community. In this case, U-report could continue the current institutional arrangement, being managed by UNICEF. While this would create a dependency situation in terms of funding, the recurring costs are minimal and could likely be covered by active fundraising. Moreover, a UNICEF-based 
arrangement would enable all potential beneficiaries to propose different polls and information campaigns, including NGOs and the government, with the flexibility to address specific requests, such as from parliamentarians, without endangering the independence and reliability of the generated information.

Irrespective of the institutional arrangement selected, a more comprehensive analysis of the longer-term objectives of U-report should be carried out to pinpoint the type of institutional and financial arrangements that would be most appropriate.

\section{Replicability}

This promising technology has already attracted the interest of other UNICEF COs worldwide. U-report is currently being rolled out in Burundi, Democratic Republic of Congo, Mozambique, South Sudan and Zimbabwe, and has already launched in Zambia, with Nepal another potential candidate. This partially reflects the fact that the initial investment used to develop the technology has already been incurred, with the open source software available for free and readily customisable. As a result, virtually any country that has wide mobile phone penetration and an interest in offering a channel for youth to voice their opinion stands to benefit. The only major requisites are having a capable project manager, a strong network of youth-oriented NGOs who would be willing to partner in the initiative, and seed money for awareness campaigns to promote U-report and sign up recruits.

\section{Findings and recommendations}

This initiative is very dynamic and the approach is still evolving on the basis of lessons learned.

All U-reporters interviewed were very enthusiastic and felt that U-report held great promise to give them a voice, which is the main objective of the tool. Most would like the system to offer more opportunities to dialogue directly with each other as well as with their district officials and parliamentarians. Specific mechanisms suggested for achieving this included: (i) the creation of U-report Chapters or Clubs; (ii) holding district U-report events; and (iii) engaging U-reporters more actively in identifying and articulating poll questions.

As noted in several discussions, Facebook could be a very useful channel to give youth with internet access the opportunity to engage with each other through the U-report system. While it could increase exposure, encourage additional participation and generate more information, a U-report Facebook page could also provide a useful platform for disseminating results and increasing the breadth of outreach activities. However, management has so far resisted calls for creating a U-report Facebook page. This reflects the fact that internet penetration in Uganda is less than 10 per cent of the population and mostly restricted to those of wealthy means, which is not the case for mobile phones. It is also important to recognise that less than 2 per cent of Uganda's population uses Facebook. Given these realities, any additional input on Facebook that directly influenced the U-report poll results would create an unwanted bias in favour of wealthier youth who enjoy internet access. Following these considerations, a U-report Twitter account was created to expand participatory opportunities, which appears a sensible strategy to further the conversation and debate without adversely affecting the poll results.

The group process observed by the mission for the formulation of poll questions appeared to be very effective, but the process of finalising questions in person and via email was less so. Several reporters commented that the polls were sometimes phrased in ways that made it difficult to understand what was being asked. More U-reporter involvement in formulating questions could help minimise this problem, and concerted efforts are being directed to overcome this challenge.

In terms of inclusivity, it was frequently mentioned that the voices of children are being captured through patrons who send in messages on their behalf. However, such messages are not currently identifiable in the database as coming from children, so it is not possible to know what the views of children reporters actually are. The question of whether adults should be allowed to participate was raised and discussed, with the conclusion that many adults have useful views on burning issues that affect youth, and their voices should also be heard. In addition to finding an appropriate way to incorporate adult opinions, it will be important to expand the number of languages in which poll questions are transmitted to maximise the reach and inclusivity of U-report, especially into rural areas. 
Regarding the institutionalisation of U-report, the current plan is to work towards eventually transferring responsibility to a consortium of NGO partners. As discussed earlier, however, this may not be realistic. As a result, it may be strategic for UNICEF Uganda to retain control of U-report over the medium term and consider creating a core staff position to continue to manage the initiative. Encouraging partner organisations to volunteer to host district activities, to create district platforms on the U-report webpage and to develop district polls on issues of local interest could further support institutionalisation efforts.

Other possibilities to facilitate greater involvement of U-reporters, protect identities and ensure that the voices of the most vulnerable are better heard include:

- Using an opt-in procedure in the registration process that gives reporters the choice to allow or not allow their names to be used in media campaigns;

- Adding a messaging application to the U-report website that can only be used by persons registering with a nickname;

- Establishing a number of small subgroups in which U-reporters in Kampala could also be invited to participate and divide the work of formulating poll questions among them; districts wishing to submit poll questions specific to their districts could do the same thing;

- Never releasing the name of a U-reporter without first asking for permission at time of use;

- Establishing transparent criteria for selecting those to be acknowledged and interviewed;

- Implementing proactive awareness campaigns in vulnerable areas to register U-reporters;

- Continuing the current practice of allowing persons of any age to register;

- Encouraging community reporters to report messages that children want to send, and adding an element in the polling format to indicate whether a message is sent on one's own behalf or on behalf of someone else, and if the latter, the age of the person who originated the message;

- Translating poll questions, messages and findings into multiple languages.

\section{Utility of RapidSMS tools for monitoring}

The SMS-based innovations that are being rolled out through UNICEF Uganda are providing the opportunity to strengthen existing country systems and processes, as well as creating the demand for quality data that can be used and acted upon by relevant stakeholders, including youth. In particular, given that the quality in terms of timeliness and accuracy of currently available reporting and information systems is variable and coverage is limited, RTM innovations have a crucial future role.

The range of SMS applications being developed by UNICEF Uganda's T4D team are well suited for gathering information that is easy to collect, sensitive to change and actionable. While they are based on an Open Source platform that can be easily deployed in other countries, T4D innovations also have the potential to be applied to all sectors and across UNICEF's programmatic areas. The data from these RTM initiatives have already become an important source of information for advocacy and action in Uganda and led to tangible, positive results. Moreover the relevance of T4D real-time innovations for tracking and verifying a wide range of indicators has already been established, further enabling the UNICEF CO (and government) to monitor progress at the subnational level (both district and community).

It is important, however, to emphasise that the different SMS RTM tools only provide an indication or 'pulse' of social conditions and must be triangulated with other more robust data sources, such as surveys (DHS, MICS, censuses, etc.) and other ad hoc rapid assessments and/or qualitative data, to fill critical data gaps and validate, as needed, the information provided from routine sources. 


\section{Notes}

1 For U-report, these included visits to: (i) the Girls' Education Movement (GEM)

headquarters located in Kampala and the

Church of Uganda venue in Gomba, where focus group discussions were held with selected U-reporters, (ii) a GEM introductory training session in Mpigi, and (iii) a GEM school in Kyegegwa District, where the mission met with school officials and students who had benefited from the Back-to-School initiative that grew out of a U-report poll. For
mTrac, these included visits to District Health Offices and various health facilities in Gomba, Mpigi and Kyegegwa Districts, as well as an interview with a Village Health Team (VHT) Coordinator in Mpigi District.

2 Findings reported here reflect mainly views expressed by biostatisticians who manage the mTrac system in three district health offices and persons in health facilities at different levels who transmit information via SMS to the districts. 